\title{
El cabildo catedral de León : clero, monarquía y sociedad (1073-1289)
}

Thèse de doctorat en histoire médiévale, sous la direction de Carlos M. Reglero de la Fuente, université de Valladolid, soutenue le 29 novembre 2019

\section{Alberto Navarro Baena}

\section{(2) OpenEdition}

1 Journals

\section{Édition électronique}

URL : https://journals.openedition.org/cem/17308

DOI : $10.4000 /$ cem. 17308

ISSN : 1954-3093

\section{Éditeur}

Centre d'études médiévales Saint-Germain d'Auxerre

\section{Référence électronique}

Alberto Navarro Baena, «El cabildo catedral de León : clero, monarquía y sociedad (1073-1289) », Bulletin du centre d'études médiévales d'Auxerre | BUCEMA [En ligne], 24.1 | 2020, mis en ligne le 21 septembre 2020, consulté le 22 septembre 2022. URL : http://journals.openedition.org/cem/17308 ; DOI : https://doi.org/10.4000/cem.17308

\section{Ce document a été généré automatiquement le 22 septembre 2022}

Creative Commons - Attribution - Pas d'Utilisation Commerciale - Partage dans les Mêmes Conditions 4.0 International - CC BY-NC-SA 4.0

https://creativecommons.org/licenses/by-nc-sa/4.0/ 


\section{El cabildo catedral de León : clero, monarquía y sociedad (1073-1289)}

Thèse de doctorat en histoire médiévale, sous la direction de Carlos M. Reglero de la Fuente, université de Valladolid, soutenue le 29 novembre 2019

\section{Alberto Navarro Baena}

1 Cette thèse porte sur le chapitre de chanoines de l'église cathédrale de León au Moyen Âge et ses rapports avec le pouvoir, le territoire et la société. Pour réaliser cette étude nous avons fait appel à l'approche prosopographique. Le chapitre de León avait déjà fait l'objet d'une étude institutionnelle publiée par Tomás Villacorta en $1974^{1}$, qui couvrait toute la période allant du $\mathrm{XII}^{\mathrm{e}}$ au $\mathrm{XIX}^{\mathrm{e}}$ siècle. Cependant, le manque de documents de type institutionnel concernant les $\mathrm{XII}^{\mathrm{e}}$ et $\mathrm{XIII}^{\mathrm{e}}$ siècles n'avait permis de traiter ces deux siècles que sommairement. Par la suite, d'autres travaux se rapportant au chapitre de León ont été publiés, mais ils portaient essentiellement sur le bas Moyen Âge ${ }^{2}$. L'histoire du chapitre au milieu du Moyen Âge n'avait donc jamais été étudiée en profondeur.

2 La thèse s'appuie sur une étude minutieuse des sources documentaires. Le chapitre de León est un cas privilégié en ce qui concerne les sources relatives au Moyen Âge central, car les archives de la cathédrale de León en ont conservé, jusqu'à aujourd'hui, 
un nombre bien plus important que celles dont disposent d'autres cathédrales des royaumes occidentaux d'Espagne pour la même période. Dès le milieu du XI ${ }^{\mathrm{e}}$ siècle, il existe des documents diplomatiques et nécrologiques se référant à divers membres du chapitre cathédral, ce qui facilite la tâche de l'historien. Ceci nous permet, par exemple, de savoir comment a évolué le nombre de chanoines entre 1073 et 1289. Toute la documentation diplomatique antérieure à 1300 est éditée dans la Colección documental del archivo de la catedral de León ${ }^{3}$, mais nous nous sommes également attachés à analyser la structure et le contenu des divers cartulaires où ces actes ont été consignés, ainsi que d'autres manuscrits léonais réalisés pour la plupart à l'époque étudiée.

3 Les archives de Léon comportent ainsi trois cartulaires médiévaux. Le Tumbo Legionense, contenant un millier de documents, est le plus ancien et le plus volumineux. Compilé au début du XII ${ }^{e}$ siècle, autour de l'an 1124, il est structuré en deux parties: la documentation cathédrale proprement dite et les diplômes de divers monastères dont les patrimoines ont été réunis à celui de la cathédrale. Cette structure reflète l'organisation des archives lors de la compilation, elle-même liée à la lutte que mène le diocèse de León pour conserver le privilège d'exemption du métropolitain face aux prétentions de l'archevêque de Tolède. Le deuxième cartulaire est le Libro de las Estampas, compilé à la fin du XII siècle à partir du Tumbo. Il rassemble vingt-cinq documents, essentiellement des donations royales, et des miniatures de divers monarques et de la comtesse doña Sancha. Il s'agit d'une aristocrate qui, vers 1045, a donné le monastère San Antolín del Esla à la cathédrale de León, ce qui a provoqué son assassinat par son neveu. Le dernier cartulaire médiéval est le Libro de los Testamentos, élaboré au milieu du Xve siècle, qui renferme quarante-sept testaments de membres du chapitre dont ne fait mention aucune autre source. Citons, comme exemple significatif, celui du chanoine Fernando Gutiérrez, de 1213, l'un des rares à permettre l'étude du concubinage des clercs.

4 Le Becerro de Presentaciones est un autre codex important ${ }^{4}$. Il s'agit d'un manuscrit décrivant l'organisation territoriale du diocèse, recensant toutes les églises qui devaient payer des rentes à l'Église de León, par archidiaconats et archiprêtrés. Bien qu'ayant été copié en 1468, il est indiqué dans le manuscrit qu'il s'agit d'une copie du becerro antiguo, dont la datation n'est pas précisée. Cependant, l'étude prosopographique de la soixantaine de clercs mentionnée permet de conclure qu'il fut réalisé autour de 1255, au début du pontificat de l'évêque Martín Fernández (1254-1289).

5 En dernier lieu, nous avons analysé les six manuscrits nécrologiques médiévaux conservés aux archives de la cathédrale ${ }^{5}$. Il s'agit d'une particularité léonaise, car dans d'autres cathédrales ils ont disparu ou nous sont parvenus en très petit nombre. Tous ont été composés entre la fin du XII et la fin du XIII ${ }^{\mathrm{e}}$ siècle et couvrent quatre générations. Les personnes inscrites sont essentiellement des clercs appartenant au chapitre, mais aussi quelques laïcs, souvent apparentés aux précédents. Outre la mention de l'obit, les obituaires renseignent sur les biens donnés par le défunt et sur la commémoration des anniversaires. Tout ceci est très précieux pour connaître l'emprise du chapitre sur la ville et le diocèse de León.

6 Parallèlement à ces sources, nous avons analysé diverses inscriptions épigraphiques. La cathédrale de León conserve un bon nombre d'inscriptions, dont la datation s'échelonne entre le $\mathrm{IX}^{\mathrm{e}}$ et le $\mathrm{xx}^{\mathrm{e}}$ siècle. Une cinquantaine est antérieure à l'année 1300 et plus de la moitié de ces épigraphes date du milieu du xiII ${ }^{e}$ siècle. Il s'agit 
essentiellement d'inscriptions funéraires, qui fournissent parfois des informations exclusives à d'autres sources, complétant celles qui figurent dans les obituaires.

7 À partir de cette documentation, nous avons étudié les clercs du chapitre de León en utilisant la méthode prosopographique. Nous avons créé une base de données à partir des obituaires, car ils consignent les noms de pratiquement tous les membres du chapitre de la période étudiée, soit 440 individus au total. La plupart de ces clercs figurent aussi dans les diplômes de la cathédrale de León. Nous avons ainsi pu établir l'origine des chanoines. Ils étaient principalement issus de la société léonaise, comme en témoignent leurs patronymes et leurs liens avec des familles locales. Il est impossible de connaître l'ascendance de la plupart d'entre eux, surtout au XII siècle. En revanche, on observe que beaucoup avaient des ancêtres francs, arrivés à León en empruntant le chemin de Saint-Jacques-de-Compostelle, comme en témoigne l'insigne officier royal don Albertino (1123-1164), dont le fils Juan Albertino devint évêque. Pendant son épiscopat (1140-1181), on observe une augmentation du nombre d'archidiacres d'origine franque, qui devaient provenir de la communauté établie à León et qui ont probablement été désignés par lui. Un autre aspect étudié concerne la carrière ecclésiastique. Les données nécrologiques et diplomatiques permettent de reconstituer le cursus de chaque membre du chapitre. Nous notons ainsi, dans leurs trajectoires, que plusieurs sont restés chanoines tandis que d'autres ont été archidiacres voire évêques. La méthode prosopographique a également permis de découvrir les relations de parenté des chanoines avec des figures importantes, comme les rois et les nobles de León, ou encore les chevaliers et les boni homines de la ville.

La thèse se subdivise en trois parties axées autour de trois dates clés de l'histoire du siège épiscopal de León. La première correspond à la restauration de l'évêque Pelayo (1065-1085), marquée par la consécration de la cathédrale en novembre 1073. À partir de cette date, le clergé de la cathédrale apparaît régulièrement dans la documentation, ce qui permet de l'étudier. La deuxième période coïncide avec l'abandon de la vie communautaire décidé en 1120 par l'évêque Diego (1112-1130), qui se traduit par la cession de bénéfices ecclésiastiques à chaque chanoine et par la décadence de la vie en communauté. La troisième correspond à la réforme du chapitre mise en œuvre par le cardinal Pelayo Albanense (1206-1230) et sanctionnée par Honorius III en 1224. Les constitutions édictées par ce cardinal furent observées par le chapitre jusqu'au $\mathrm{xVIII}^{\mathrm{e}}$ siècle. Ayant promu l'assemblée capitulaire et permis une nouvelle répartition des canonicats au détriment des biens de la rente épiscopale, elles se sont traduites par une perte de pouvoir de l'évêque et un renforcement de celui du chapitre. Ces constitutions sont violées à la fin du XIII e siècle par l'évêque Martín Fernández qui s'approprie certains biens du chapitre. Toutefois, après la mort du prélat en 1289, les chanoines réussissent à récupérer leurs propriétés et à faire valoir à nouveau les constitutions. Notre étude s'arrête à ce moment.

Le clergé cathédral constitue l'axe central de la thèse. La présence d'une communauté cléricale dans la cathédrale de León débute au IX $\mathrm{I}^{\mathrm{e}}$ siècle, après la prise de la ville par Ordoño I des Asturies (856). Néanmoins, peu de documents antérieurs au milieu du $\mathrm{XI}^{\mathrm{e}}$ siècle permettent d'étudier ces clercs. Il s'agit d'une communauté constituée d'hommes désignés sous le terme générique de monacos, identifiés parfois dans les sources comme diacres, prêtres et abbés. Dès l'année 1073, on sait qu'ils sont une trentaine de clercs. Cinquante ans plus tard, ils sont déjà quarante, sans tenir compte des archidiacres, comme le précise l'évêque Diego en 1120. Un siècle plus tard, la 
communauté s'est considérablement accrue. Le cardinal Albanense fixe le nombre de chanoines majeurs à cinquante, et le nombre de portionarii à vingt-cinq, imposant ainsi un maximum de soixante-quinze bénéficiaires.

Cet accroissement au cours du $\mathrm{XII}^{\mathrm{e}}$ siècle est la conséquence de la concession par l'évêque d'un nombre excessif de prébendes au point qu'elles finissent par surpasser les ressources de l'Église de León et faire chuter les bénéfices. C'est pourquoi, en 1224, l'Albanense limite le nombre de bénéficiaires et fixe le montant des prébendes. Malgré ces règles, au milieu du XIII ${ }^{e}$ siècle, le nombre de bénéficiaires est supérieur au quota fixé, essentiellement en raison de l'intervention du pape et des cardinaux de Rome qui accordent à leurs proches des bénéfices de l'église de León.

11 Il est possible de reconstituer le statut des chanoines à partir des mentions documentaires. En 1120, l'évêque Diego distingue deux catégories: les chanoines majeurs et les chanoines mineurs. Les premiers sont des prêtres tandis que les seconds sont des diacres ou sous-diacres. Même si dans la documentation postérieure ils sont tous désignés comme chanoines, sans spécifier leur grade ecclésiastique, ceux-ci peuvent être connus grâce aux obituaires. Au XIII ${ }^{e}$ siècle, les chanoines mineurs sont appelés portionarii et le terme canonicus est réservé aux chanoines majeurs. Les canonici reçoivent le double de la rétribution des portionarii. Pendant cette période, on observe que la carrière ecclésiastique commence d'ordinaire aux échelons les plus bas. Certains chanoines sont clercs du chœur avant de devenir portionarii; beaucoup d'entre eux débutent comme portionarii. La promotion dans la hiérarchie au-delà du canonicat vers une dignité ou un archidiaconat n'est possible que pour quelques privilégiés. Pour être promu, il fallait avoir une longue ancienneté au sein du chapitre ou la faveur de certaines personnes influentes. Des chanoines parvenaient à une dignité ou à l'archidiaconat à l'aube de leur mort alors que d'autres clercs débutaient directement avec le grade d'archidiacre ou accédaient très rapidement à des dignités. L'exemple le plus caractéristique est celui de Fernando Patiño (1259-1295), neveu du maestrescuela (chancelier) Fernando Guillélmez (1253-1272), qui cumule successivement deux dignités et deux archidiaconats.

12 La relation entre le chapitre et l'évêque change après la réforme du cardinal Pelayo Albanense (1224). Auparavant, le clergé de la cathédrale jouait un rôle limité dans la prise de décisions concernant les affaires du diocèse. Les réunions se tenaient à l'évêché, où étaient traitées les grandes questions (grandes fechos) de l'Église. De même, l'évêque accordait des bénéfices à qui bon lui semblait de sorte que le chapitre était taillé à sa mesure. En revanche, les constitutions de l'Albanense donnèrent aux chanoines un rôle actif dans les assemblées et l'octroi de bénéfices. Par ailleurs, au milieu du XIII siècle, des clercs non choisis par l'évêque obtiennent directement des prébendes de Rome, ce qui fait apparaitre diverses factions aux intérêts divergents au sein du chapitre. C'est la raison pour laquelle les désignations des évêques par les chanoines au cours du XIII ${ }^{\mathrm{e}}$ siècle sont souvent polémiques avec deux candidats qui se disputent la mitre. L'accroissement des biens du chapitre, décidé par le cardinal Pelayo, a également altéré la relation entre le chapitre et l'évêque. Les biens épiscopaux ont beaucoup diminué et les évêques ne parviennent pas à résoudre ce problème. Cette situation est à l'origine du litige entre l'évêque et le chapitre autour des biens capitulaires à la fin du XIII ${ }^{e}$ siècle, comme nous l'avons explicité ci-dessus.

13 Autre thème abordé : le rapport du clergé de la cathédrale avec la monarchie. Dès la création du diocèse sous le roi Ordoño I (850-866), les évêques sont issus de l'entourage 
royal comme en témoignent les documents les plus anciens. En revanche, il est difficile d'affirmer qu'il en est de même avec le clergé de la cathédrale. Les prélats n'en étaient pas nécessairement issus. $\mathrm{Au} \mathrm{x}^{\mathrm{e}}$ siècle, certains provenaient de divers monastères et, au milieu du xi ${ }^{e}$ siècle, l'évêque Pelayo, lui-même originaire de Compostelle, fut le disciple de Cresconius d'Iria (1048-1066).

Ce n'est que sous le règne d'Alphonse VI (1065-1109) que des liens entre le monarque et le chapitre apparaissent. Premier monarque à s'adresser aux chanoines dans un document les concernant exclusivement, c'est sous son règne qu'apparaissent certains clercs de la cathédrale désignés comme presbiter regis ou clericus regis, donc rattachés au roi. Cette nouveauté va perdurer. On l'observe surtout après l'abandon de la vie communautaire (1120). La reine Urraca (1109-1126), tout comme l'empereur Alphonse VII (1126-1157), est proche de quelques membres du chapitre. Ce phénomène est particulièrement évident sous Ferdinand II (1157-1188), dont relèvent certains hauts dignitaires et archidiacres, de même que sous Alphonse IX (1188-1230). De plus, certains de ces chanoines sont rattachés précisément à la chancellerie royale. Après l'unification des royaumes de León et de Castille (1230), les liens entre la famille royale et le chapitre se poursuivent. Avec Ferdinand III (1230-1252), il n'y en a aucuns, mais ils existent avec ses successeurs Alphonse X (1252-1284) et Sanche IV (1284-1295). Somme toute, celui qui exerçait une influence sur le chapitre avait son mot à dire dans le choix de l'évêque.

Ces connexions entre la monarchie et le chapitre expliquent le rôle important qu'il joue en matière judiciaire. Dans les dernières décennies du XII siècle, à la fin du règne de Ferdinand II, il est fait mention dans la documentation de quelques clercs en qualité de juges. Cette réalité est attestée par une perquisition effectuée en 1266 dans le cadre de la dispute survenue entre l'Église et la municipalité (concejo) à propos du juge ecclésiastique. Les témoins, remontant aux premières années du XIII siècle, signalent que traditionnellement il y avait toujours eu quatre juges à León, dont l'un était désigné par l'évêque et ratifié par le roi. Néanmoins, il existait aussi le juge du Livre (le Fuero Juzgo) chargé de trancher les recours. Au temps d'Alphonse IX, ce juge était Fernando Alfonso (1202-1230), chanoine du chapitre et clerc du roi. Lorsque le roi et le chanoine moururent, en l'absence de toute décision royale en la matière, cette charge fut dévolue au juge ecclésiastique, de sorte que l'Église de León s'arrogea le pouvoir d'arbitrer les procès ordinaires et les recours. Cette prééminence fit naître les plaintes du concejo qui ne parvint à obtenir le soutien du roi qu'au début du XIV siècle. Durant tout le XIII ${ }^{e}$ siècle, le rôle de l'Église en matière judiciaire fut décisif dans la ville grâce à ses liens avec la monarchie.

En dernier lieu, un autre point important abordé concerne la relation entre le clergé de la cathédrale et la société de son temps. L'Église et le chapitre de León, en tant que pouvoirs ecclésiastiques suprêmes de la ville et du diocèse, eurent un grand poids social au milieu du Moyen Âge. Leur domination territoriale s'accrut au milieu du XI $\mathrm{x}^{\mathrm{e}}$ siècle à la suite des réformes du concile de Coyanza (1055). Divers nobles firent alors des donations de biens et de rentes à l'évêque. Ainsi, le comte Diego Ansúrez († ca. 1083) fit don de tous ses biens au siège épiscopal après sa mort au combat et fut enterré dans la cathédrale.

17 À la période étudiée, l'une des familles les plus insignes était celle du comte Martín Flaínez, dont le nom apparaît, avec celui de sa mère doña Toda, dans une donation à l'évêque Pelayo en 1066. Le nécrologe de la cathédrale et les obituaires successifs se 
réfèrent au comte et à son fils, à la date du 29 mai, jour de la bataille d'Uclés (1108) au cours de laquelle il périt. Son épouse, la comtesse Sancha $(\dagger 1114)$, y figure également, de même que leurs deux fils, les comtes Rodrigo (1126-1138) et Osorio Martínez (1130-1160). Les descendants de ce dernier, dont sont issus les membres de la famille Villalobos, sont également représentés jusqu’à la fin du XIII siècle, époque à laquelle ils deviennent chanoines. De fait, au milieu du XIII ${ }^{\mathrm{e}}$ siècle, on constate parmi les membres du chapitre la présence de personnes issues de la lignée Villalobos.

En conclusion, le chapitre de la cathédrale de León connaît d'importants changements au cours de la période étudiée. Au niveau institutionnel, il passe d'une petite communauté de trente clercs à une communauté de soixante-quinze membres; et de l'exercice de la vie communautaire à la possession individuelle de ses propres biens. Au $\mathrm{XII}^{\mathrm{e}}$ siècle, l'évêque leur assure la possession de monastères urbains et diocésains. Le chapitre devient ainsi un centre de pouvoir dans lequel les plus importantes familles locales placent certains de leurs membres. Avec la réforme de 1224, les chanoines acquièrent un plus grand rôle dans l'administration du diocèse, car ils commencent à participer à la prise de décisions par le biais des réunions du chapitre. Cela leur permet de s'opposer à l'évêque, et même de gagner un procès contre lui pour la défense de leurs propriétés. Ainsi, à la fin $\mathrm{du} \mathrm{xIII}^{\mathrm{e}}$ siècle, ils ont consolidé leur pouvoir face aux éventuelles ingérences épiscopales.

Reçu : 12 mars 2020 - Accepté : 25 mai 2020

\section{NOTES}

1. T. VILLACORTA RODRíGueZ, El Cabildo Catedral de León : estudio histórico-jurídico, siglo XII-XIX, León, 1974 (Fuentes y estudios de Historia leonesa, 12).

2. Par exemple H. CASADO ALONSO, La propiedad eclesiástica en la ciudad de Burgos en el siglo XV. El cabildo catedralicio, Valladolid, 1980 ; F. J. FERNÁNDEZ CONDE, El señorío del cabildo ovetense. Estructuras agrarias de Asturias en el Tardo Medievo, Oviedo, 1994 ; M. J. LOP OTíN, El cabildo catedralicio de Toledo en el siglo XV. Aspectos institucionales y sociológicos, Madrid, 2003. Voir aussi M. N. MUNSURI ROSADO, «Clero e Iglesia en la Edad Media hispánica. Estado de la cuestión », eHumanista. Journal of Iberian Studies, 10 (2008), p. 139-169.

3. Colección documental del Archivo de la Catedral de León (775-1474), éd. E. SÁEZ, C. SÁEZ SÁNCHEZ, J. M. RUIZ ASENCIO, J. M. FERNÁNDEZ CATÓN, J. A. MARTíN FUERTES, M. HERRERO JIMÉNEZ et C. ÁLVAREZ ÁLVAREZ, 12 vol., León, 1987-2002 (Fuentes y estudios de Historia leonesa, 41-47, 54-56 et 59-60).

4. J. A. FERNÁNDEZ FLóREZ, « El "Becerro de Presentaciones". Códice 13 del Archivo de la Catedral de León. Un parroquial leonés de los siglos XIII-XV », León y su historia. Miscelánea Histórica, V, León, 1984, p. 263-565.

5. M. HERRERO JIMÉNEZ, Colección documental del Archivo de la Catedral de León, X, Obituarios medievales, León, 1994. 


\section{AUTEUR}

\section{ALBERTO NAVARRO BAENA}

Université de Valladolid 